\title{
Some generalization of integral inequalities for twice differentiable mappings involving fractional integrals
}

\author{
Mehmet Zeki Sarikaya \\ Department of Mathematics, \\ Faculty of Science and Arts, \\ Duzce University, Turkey \\ email: sarikayamz@gmail.com
}

\author{
Huseyin Budak \\ Department of Mathematics, \\ Faculty of Science and Arts, \\ Duzce University, Turkey \\ email: hsyn.budak@gmail.com
}

\begin{abstract}
In this paper, a general integral identity involving RiemannLiouville fractional integrals is derived. By use this identity, we establish new some generalized inequalities of the Hermite-Hadamard's type for functions whose absolute values of derivatives are convex.
\end{abstract}

\section{Introduction}

The following definition for convex functions is well known in the mathematical literature:

The function $f:[a, b] \subset \mathbb{R} \rightarrow \mathbb{R}$, is said to be convex if the following inequality holds

$$
f(\lambda x+(1-\lambda) y) \leq \lambda f(x)+(1-\lambda) f(y)
$$

for all $x, y \in[a, b]$ and $\lambda \in[0,1]$. We say that $f$ is concave if $(-f)$ is convex.

2010 Mathematics Subject Classification: 26D07, 26D10, 26D15, 26A33

Key words and phrases: Hermite-Hadamard's inequalities, Riemann-Liouville fractional integral, convex functions, integral inequalities 
Many inequalities have been established for convex functions but the most famous inequality is the Hermite-Hadamard's inequality, due to its rich geometrical significance and applications(see, e.g.,[12, p.137], [6]). These inequalities state that if $f: I \rightarrow \mathbb{R}$ is a convex function on the interval I of real numbers and $a, b \in$ I with $a<b$, then

$$
f\left(\frac{a+b}{2}\right) \leq \frac{1}{b-a} \int_{a}^{b} f(x) d x \leq \frac{f(a)+f(b)}{2} .
$$

Both inequalities hold in the reversed direction if $f$ is concave. We note that Hadamard's inequality may be regarded as a refinement of the concept of convexity and it follows easily from Jensen's inequality. Hadamard's inequality for convex functions has received renewed attention in recent years and a remarkable variety of refinements and generalizations have been found (see, for example, [6, 8, 9, 12], [14]-[16], [22], [23]) and the references cited therein.

In [16], Sarikaya et. al. established inequalities for twice differentiable convex mappings which are connected with Hadamard's inequality, and they used the following lemma to prove their results:

Lemma 1 Let $\mathrm{f}: \mathrm{I}^{\circ} \subset \mathbb{R} \rightarrow \mathbb{R}$ be twice differentiable function on $\mathrm{I}^{\circ}, \mathrm{a}, \mathrm{b} \in \mathrm{I}^{\circ}$ with $\mathrm{a}<\mathrm{b}$. If $\mathrm{f}^{\prime \prime} \in \mathrm{L}_{1}[\mathrm{a}, \mathrm{b}]$, then

$$
\begin{aligned}
& \frac{1}{b-a} \int_{a}^{b} f(x) d x-f\left(\frac{a+b}{2}\right) \\
& \quad=\frac{(b-a)^{2}}{2} \int_{0}^{1} m(t)\left[f^{\prime \prime}(t a+(1-t) b)+f^{\prime \prime}(t b+(1-t) a)\right] d t,
\end{aligned}
$$

where

$$
m(t):= \begin{cases}t^{2}, & t \in\left[0, \frac{1}{2}\right) \\ (1-t)^{2}, & t \in\left[\frac{1}{2}, 1\right] .\end{cases}
$$

Also, the main inequalities in [16], pointed out as follows:

Theorem 1 Let $\mathrm{f}: \mathrm{I} \subset \mathbb{R} \rightarrow \mathbb{R}$ be twice differentiable function on $\mathrm{I}^{\circ}$ with $f^{\prime \prime} \in \mathrm{L}_{1}[\mathrm{a}, \mathrm{b}]$. If $\left|\mathrm{f}^{\prime \prime}\right|$ is convex on $[\mathrm{a}, \mathrm{b}]$, then

$$
\left|\frac{1}{b-a} \int_{a}^{b} f(x) d x-f\left(\frac{a+b}{2}\right)\right| \leq \frac{(b-a)^{2}}{24}\left[\frac{\left|f^{\prime \prime}(a)\right|+\left|f^{\prime \prime}(b)\right|}{2}\right] .
$$

Theorem 2 Let $\mathrm{f}: \mathrm{I} \subset \mathbb{R} \rightarrow \mathbb{R}$ be twice differentiable function on $\mathrm{I}^{\circ}$ such that $\mathrm{f}^{\prime \prime} \in \mathrm{L}_{1}[\mathrm{a}, \mathrm{b}]$ where $\mathrm{a}, \mathrm{b} \in \mathrm{I}, \mathrm{a}<\mathrm{b}$. If $\left|\mathrm{f}^{\prime \prime}\right|^{\mathrm{q}}$ is convex on $[\mathrm{a}, \mathrm{b}], \mathrm{q}>1$, then

$$
\left|\frac{1}{b-a} \int_{a}^{b} f(x) d x-f\left(\frac{a+b}{2}\right)\right| \leq \frac{(b-a)^{2}}{8(2 p+1)^{1 / p}}\left[\frac{\left|f^{\prime \prime}(a)\right|^{q}+\left|f^{\prime \prime}(b)\right|^{q}}{2}\right]^{1 / q}
$$


where $\frac{1}{\mathrm{p}}+\frac{1}{\mathrm{q}}=1$.

In the following we will give some necessary definitions and mathematical preliminaries of fractional calculus theory which are used further in this paper. More details, one can consult [7, 10, 11, 13].

Definition 1 Let $\mathrm{f} \in \mathrm{L}_{1}[\mathrm{a}, \mathrm{b}]$. The Riemann-Liouville integrals $\mathrm{J}_{\mathrm{a}+}^{\alpha} \mathrm{f}$ and $\mathrm{J}_{\mathrm{b}-}^{\alpha} \mathrm{f}$ of order $\alpha>0$ with $\mathrm{a} \geq 0$ are defined by

$$
J_{a+}^{\alpha} f(x)=\frac{1}{\Gamma(\alpha)} \int_{a}^{x}(x-t)^{\alpha-1} f(t) d t, \quad x>a
$$

and

$$
\mathrm{J}_{\mathrm{b}-}^{\alpha} \mathrm{f}(\mathrm{x})=\frac{1}{\Gamma(\alpha)} \int_{x}^{\mathrm{b}}(\mathrm{t}-x)^{\alpha-1} \mathrm{f}(\mathrm{t}) \mathrm{dt}, \quad x<\mathrm{b}
$$

respectively. Here, $\Gamma(\alpha)$ is the Gamma function and $\mathrm{J}_{\mathrm{a}+}^{0} \mathrm{f}(\mathrm{x})=\mathrm{J}_{\mathrm{b}-}^{0} \mathrm{f}(\mathrm{x})=\mathrm{f}(\mathrm{x})$.

Meanwhile, Sarikaya et al. [19] presented the following important integral identity including the first-order derivative of $f$ to establish many interesting Hermite-Hadamard type inequalities for convexity functions via RiemannLiouville fractional integrals of the order $\alpha>0$.

Lemma 2 Let $\mathrm{f}:[\mathrm{a}, \mathrm{b}] \rightarrow \mathbb{R}$ be a differentiable mapping on $(\mathrm{a}, \mathrm{b})$ with $0 \leq$ $\mathrm{a}<\mathrm{b}$. If $\mathrm{f}^{\prime} \in \mathrm{L}[\mathrm{a}, \mathrm{b}]$, then the following equality for fractional integrals holds:

$$
\begin{aligned}
& \frac{2^{\alpha-1} \Gamma(\alpha+1)}{(b-a)^{\alpha}}\left[J_{\left(\frac{a+b}{2}\right)+}^{\alpha} f(b)+J_{\left(\frac{a+b}{2}\right)-}^{\alpha} f(a)\right]-f\left(\frac{a+b}{2}\right) \\
& =\frac{b-a}{4}\left\{\int_{0}^{1} t^{\alpha} f^{\prime}\left(\frac{t}{2} a+\frac{2-t}{2} b\right) d t-\int_{0}^{1} t^{\alpha} f^{\prime}\left(\frac{2-t}{2} a+\frac{t}{2} b\right) d t\right\}
\end{aligned}
$$

with $\alpha>0$.

It is remarkable that Sarikaya et al. [19] first give the following interesting integral inequalities of Hermite-Hadamard type involving Riemann-Liouville fractional integrals.

Theorem 3 Let $\mathrm{f}:[\mathrm{a}, \mathrm{b}] \rightarrow \mathbb{R}$ be a positive function with $0 \leq \mathrm{a}<\mathrm{b}$ and $\mathrm{f} \in \mathrm{L}_{1}[\mathrm{a}, \mathrm{b}]$. If $\mathrm{f}$ is a convex function on $[\mathrm{a}, \mathrm{b}]$, then the following inequalities for fractional integrals hold:

$$
f\left(\frac{a+b}{2}\right) \leq \frac{2^{\alpha-1} \Gamma(\alpha+1)}{(b-a)^{\alpha}}\left[J_{\left(\frac{a+b}{2}\right)+}^{\alpha} f(b)+J_{\left(\frac{a+b}{2}\right)-}^{\alpha} f(a)\right] \leq \frac{f(a)+f(b)}{2}
$$

with $\alpha>0$. 
For some recent results connected with fractional integral inequalities see $([1,2,3,4,5],[17],[18],[20],[21],[24])$

In this paper, we expand the Lemma 2 to the case of including a twice differentiable function involving Riemann-Liouville fractional integrals and some other integral inequalities using the generalized identity is obtained for fractional integrals.

\section{Main results}

For our results, we give the following important fractional integrtal identity:

Lemma 3 Let $\mathrm{f}:[\mathrm{a}, \mathrm{b}] \rightarrow \mathbb{R}$ be twice differentiable mapping on $(\mathrm{a}, \mathrm{b})$ with $0 \leq \mathrm{a}<\mathrm{b}$. If $\mathrm{f}^{\prime \prime} \in \mathrm{L}[\mathrm{a}, \mathrm{b}]$, then the following equality for fractional integrals holds:

$$
\begin{aligned}
& (\alpha+1)(1-\lambda)^{\alpha} \lambda^{\alpha} f(\lambda a+(1-\lambda) b) \\
& \quad-\frac{(\alpha+1) \Gamma(\alpha+1)}{(b-a)^{\alpha}}\left[\lambda^{\alpha+1} J_{(\lambda a+(1-\lambda) b)-}^{\alpha} f(a)+(1-\lambda)^{\alpha+1} J_{(\lambda a+(1-\lambda) b)+}^{\alpha} f(b)\right] \\
& =-(b-a)^{2}(1-\lambda)^{\alpha+1} \lambda^{\alpha+1}\left\{(1-\lambda) \int_{0}^{1} t^{\alpha+1} f^{\prime \prime}[t(\lambda a+(1-\lambda) b)+(1-t) a] d t\right. \\
& \left.\quad+\lambda \int_{0}^{1}(1-t)^{\alpha+1} f^{\prime \prime}[t b+(1-t)(\lambda a+(1-\lambda) b)] d t\right\}
\end{aligned}
$$

where $\lambda \in(0,1)$ and $\alpha>0$.

Proof. Integrating by parts

$$
\begin{aligned}
& \int_{0}^{1} t^{\alpha+1} f^{\prime \prime}[t(\lambda a+(1-\lambda) b)+(1-t) a] d t \\
& =\left.\frac{t^{\alpha+1} f^{\prime}[t(\lambda a+(1-\lambda) b)+(1-t) a]}{(1-\lambda)(b-a)}\right|_{0} ^{1} \\
& \quad-\frac{\alpha+1}{(1-\lambda)(b-a)} \int_{0}^{1} t^{\alpha} f^{\prime}[t(\lambda a+(1-\lambda) b)+(1-t) a] d t \\
& =\frac{f^{\prime}(\lambda a+(1-\lambda) b)}{(1-\lambda)(b-a)}-\frac{\alpha+1}{(1-\lambda)(b-a)}
\end{aligned}
$$




$$
\begin{aligned}
\times & {\left[\frac{f(\lambda a+(1-\lambda) b)}{(1-\lambda)(b-a)}-\frac{\alpha}{(1-\lambda)(b-a)} \int_{0}^{1} t^{\alpha-1} f[t(\lambda a+(1-\lambda) b)+(1-t) a] d t\right] } \\
= & \frac{f^{\prime}(\lambda a+(1-\lambda) b)}{(1-\lambda)(b-a)}-\frac{(\alpha+1) f(\lambda a+(1-\lambda) b)}{(1-\lambda)^{2}(b-a)^{2}} \\
& +\frac{(\alpha+1) \alpha}{(1-\lambda)^{\alpha+2}(b-a)^{\alpha+2}} \int_{a}^{\lambda a+(1-\lambda) b}(x-a)^{\alpha-1} f(x) d x \\
= & \frac{f^{\prime}(\lambda a+(1-\lambda) b)}{(1-\lambda)(b-a)}-\frac{(\alpha+1) f(\lambda a+(1-\lambda) b)}{(1-\lambda)^{2}(b-a)^{2}} \\
& +\frac{(\alpha+1) \Gamma(\alpha+1)}{(1-\lambda)^{\alpha+2}(b-a)^{\alpha+2}} J_{(\lambda a+(1-\lambda) b)^{-}}^{\alpha} f(a)
\end{aligned}
$$

that is,

$$
\begin{aligned}
& -\int_{0}^{1} t^{\alpha+1} f^{\prime \prime}[t(\lambda a+(1-\lambda) b)+(1-t) a] d t \\
& =-\frac{f^{\prime}(\lambda a+(1-\lambda) b)}{(1-\lambda)(b-a)}+\frac{(\alpha+1) f(\lambda a+(1-\lambda) b)}{(1-\lambda)^{2}(b-a)^{2}} \\
& \quad-\frac{(\alpha+1) \Gamma(\alpha+1)}{(1-\lambda)^{\alpha+2}(b-a)^{\alpha+2}} J_{(\lambda a+(1-\lambda) b)^{-}}^{\alpha} f(a)
\end{aligned}
$$

and similarly we have

$$
\begin{aligned}
- & \int_{0}^{1}(1-t)^{\alpha+1} f^{\prime \prime}[t b+(1-t)(\lambda a+(1-\lambda) b)] d t \\
= & \frac{f^{\prime}(\lambda a+(1-\lambda) b)}{\lambda(b-a)}+\frac{(\alpha+1) f(\lambda a+(1-\lambda) b)}{\lambda^{2}(b-a)^{2}} \\
& -\frac{(\alpha+1) \alpha}{\lambda^{\alpha+2}(b-a)^{\alpha+2}} \int_{\lambda a+(1-\lambda) b}^{b}(b-x)^{\alpha-1} f(x) d x \\
= & \frac{f^{\prime}(\lambda a+(1-\lambda) b)}{\lambda(b-a)}+\frac{(\alpha+1) f(\lambda a+(1-\lambda) b)}{\lambda^{2}(b-a)^{2}} \\
& -\frac{(\alpha+1) \Gamma(\alpha+1)}{\lambda^{\alpha+2}(b-a)^{\alpha+2}} J_{(\lambda a+(1-\lambda) b)^{+}}^{\alpha} f(b) .
\end{aligned}
$$

Adding (8) and (9) we have (7). This completes the proof. 
Corollary 1 Under the assumptions Lemma 3 with $\lambda=\frac{1}{2}$, then it follows that

$$
\begin{aligned}
& \frac{-(b-a)^{2}}{8}\left\{\int_{0}^{1} t^{\alpha+1} f^{\prime \prime}\left[t\left(\frac{a+b}{2}\right)+(1-t) a\right] d t\right. \\
& \left.+\int_{0}^{1}(1-t)^{\alpha+1} f^{\prime \prime}\left[t b+(1-t) \frac{a+b}{2}\right] d t\right\} \\
& =(\alpha+1) f\left(\frac{a+b}{2}\right)-\frac{(\alpha+1) \Gamma(\alpha+1)}{(b-a)^{\alpha} 2^{1-\alpha}}\left[J_{\left(\frac{a+b}{2}\right)^{-}}^{\alpha}(a)+J_{\left(\frac{a+b}{2}\right)^{+}}^{\alpha} f(b)\right] .
\end{aligned}
$$

Remark 1 If we choose $\alpha=1$ in Corollary 1, we have

$$
\begin{aligned}
& f\left(\frac{a+b}{2}\right)-\frac{1}{b-a} \int_{a}^{b} f(x) d x \\
& =\frac{-(b-a)^{2}}{16}\left\{\int_{0}^{1} t^{2} f^{\prime \prime}\left[t\left(\frac{a+b}{2}\right)+(1-t) a\right] d t\right. \\
& \left.\quad+\int_{0}^{1}(1-t)^{2} f^{\prime \prime}\left[t b+(1-t) \frac{a+b}{2}\right] d t\right\} .
\end{aligned}
$$

Theorem 4 Let $f:[a, b] \rightarrow \mathbb{R}$ be twice differentiable mapping on $(a, b)$ with $0 \leq \mathrm{a}<\mathrm{b}$. If $\left|\mathrm{f}^{\prime \prime}\right|^{\mathrm{q}}, \mathrm{q} \geq 1$ is convex on $[\mathrm{a}, \mathrm{b}]$, then the following inequality for fractional integrals holds:

$$
\begin{aligned}
& \mid(\alpha+1)(1-\lambda)^{\alpha} \lambda^{\alpha} f(\lambda a+(1-\lambda) b)-\frac{(\alpha+1) \Gamma(\alpha+1)}{(b-a)^{\alpha}} \\
& \times\left[\lambda^{\alpha+1} J_{(\lambda a+(1-\lambda) b)^{-}}^{\alpha} f(a)+(1-\lambda)^{\alpha+1} J_{(\lambda a+(1-\lambda) b)^{+}}^{\alpha} f(b)\right] \mid \\
& \leq \frac{(b-a)^{2}(1-\lambda)^{\alpha+1} \lambda^{\alpha+1}}{(\alpha+2)^{1-\frac{1}{q}}}\left\{(1-\lambda)\left(\frac{(\alpha+2)\left|f^{\prime \prime}(\lambda a+(1-\lambda) b)\right|^{q}+\left|f^{\prime \prime}(a)\right|^{q}}{\alpha+3}\right)^{\frac{1}{q}}\right. \\
& \left.\quad+\lambda\left(\frac{(\alpha+2)\left|f^{\prime \prime}(\lambda a+(1-\lambda) b)\right|^{q}+\left|f^{\prime \prime}(b)\right|^{q}}{\alpha+3}\right)^{\frac{1}{q}}\right\} .
\end{aligned}
$$

where $\lambda \in(0,1)$ and $\alpha>0$. 
Proof. Firstly, we suppose that $q=1$. Using Lemma 3 and convexity of $\left|f^{\prime \prime}\right|^{q}$, we find that

$$
\begin{aligned}
& \mid(\alpha+1)(1-\lambda)^{\alpha} \lambda^{\alpha} f(\lambda a+(1-\lambda) b)-\frac{(\alpha+1) \Gamma(\alpha+1)}{(b-a)^{\alpha}} \\
& \quad \times\left[\lambda^{\alpha+1} J_{\left.(\lambda a+(1-\lambda) b)^{\alpha}-f(a)+(1-\lambda)^{\alpha+1} J_{(\lambda a+(1-\lambda) b)+}^{\alpha} f(b)\right] \mid}^{1} \leq(b-a)^{2}(1-\lambda)^{\alpha+1} \lambda^{\alpha+1}\left\{(1-\lambda) \int_{0}^{1} t^{\alpha+1}\left|f^{\prime \prime}[t(\lambda a+(1-\lambda) b)+(1-t) a]\right| d t\right.\right. \\
& \left.\quad+\lambda \int_{0}^{1}(1-t)^{\alpha+1}\left|f^{\prime \prime}[t b+(1-t)(\lambda a+(1-\lambda) b)]\right| d t\right\} \\
& \leq(b-a)^{2}(1-\lambda)^{\alpha+1} \lambda^{\alpha+1}\left\{(1-\lambda) \int_{0}^{1} t^{\alpha+1}\left[t\left|f^{\prime \prime}(\lambda a+(1-\lambda) b)\right|+(1-t)\left|f^{\prime \prime}(a)\right|\right] d t\right. \\
& \left.\quad+\lambda \int_{0}^{1}(1-t)^{\alpha+1}\left[t\left|f^{\prime \prime}(b)\right|+(1-t)\left|f^{\prime \prime}(\lambda a+(1-\lambda) b)\right|\right] d t\right\} \\
& =\frac{(b-a)^{2}(1-\lambda)^{\alpha+1} \lambda^{\alpha+1}}{\alpha+2}\left\{(1-\lambda)\left(\frac{(\alpha+2)\left|f^{\prime \prime}(\lambda a+(1-\lambda) b)\right|+\left|f^{\prime \prime}(a)\right|}{\alpha+3}\right)\right. \\
& \left.+\lambda\left(\frac{(\alpha+2)\left|f^{\prime \prime}(\lambda a+(1-\lambda) b)\right|+\left|f^{\prime \prime}(b)\right|^{q}}{\alpha+3}\right)\right\} \cdot
\end{aligned}
$$

Secondly, we suppose that $q>1$. Using Lemma 3 and power mean inequality, we have

$$
\begin{aligned}
& \left\{(1-\lambda) \int_{0}^{1} t^{\alpha+1} f^{\prime \prime}[t(\lambda a+(1-\lambda) b)+(1-t) a] d t\right. \\
& \left.+\lambda \int_{0}^{1}(1-t)^{\alpha+1} f^{\prime \prime}[t b+(1-t)(\lambda a+(1-\lambda) b)] d t\right\} \\
& \leq(1-\lambda)\left(\int_{0}^{1} t^{\alpha+1}\right)^{1-\frac{1}{q}}\left(\int_{0}^{1} t^{\alpha+1}\left|f^{\prime \prime}[t(\lambda a+(1-\lambda) b)+(1-t) a]\right|^{q} d t\right)^{\frac{1}{q}} \\
& +\lambda\left(\int_{0}^{1}(1-t)^{\alpha+1}\right)^{1-\frac{1}{q}}\left(\int_{0}^{1}(1-t)^{\alpha+1}\left|f^{\prime \prime}[t b+(1-t)(\lambda a+(1-\lambda) b)]\right|^{q} d t\right)^{\frac{1}{q}} .
\end{aligned}
$$


Hence, using convexity of $\left|f^{\prime \prime}\right|^{q}$ and (11) we obtain

$$
\begin{aligned}
& \mid(\alpha+1)(1-\lambda)^{\alpha} \lambda^{\alpha} f(\lambda a+(1-\lambda) b)-\frac{(\alpha+1) \Gamma(\alpha+1)}{(b-a)^{\alpha}} \\
& \quad \times\left[\lambda^{\alpha+1} J_{(\lambda a+(1-\lambda) b)-}^{\alpha} f(a)+(1-\lambda)^{\alpha+1} J_{(\lambda a+(1-\lambda) b)+}^{\alpha} f(b)\right] \mid \\
& \leq \frac{(b-a)^{2}(1-\lambda)^{\alpha+1} \lambda^{\alpha+1}}{(\alpha+2)^{1-\frac{1}{q}}}\left\{(1-\lambda)\left(\int_{0}^{1} t^{\alpha+1}\left[t\left|f^{\prime \prime}(\lambda a+(1-\lambda) b)\right|+(1-t)\left|f^{\prime \prime}(a)\right|\right] d t\right)^{\frac{1}{q}}\right. \\
& \quad+\lambda\left(\int_{0}^{1}(1-t)^{\alpha+1}\left[t\left|f^{\prime \prime}(b)\right|+(1-t)\left|f^{\prime \prime}(\lambda a+(1-\lambda) b)\right| d t\right)^{\frac{1}{q}}\right\} \\
& \leq \frac{(b-a)^{2}(1-\lambda)^{\alpha+1} \lambda^{\alpha+1}}{(\alpha+2)^{1-\frac{1}{q}}}\left\{(1-\lambda)\left(\frac{(\alpha+2)\left|f^{\prime \prime}(\lambda a+(1-\lambda) b)\right|+\left|f^{\prime \prime}(a)\right|}{(\alpha+2)(\alpha+3)}\right)^{\frac{1}{q}}\right. \\
& \left.\quad+\lambda\left(\frac{(\alpha+2)\left|f^{\prime \prime}(\lambda a+(1-\lambda) b)\right|+\left|f^{\prime \prime}(b)\right|^{q}}{(\alpha+2)(\alpha+3)}\right)^{\frac{1}{q}}\right\} .
\end{aligned}
$$

This completes the proof.

Corollary 2 Under assumption Theorem 4 with $\lambda=\frac{1}{2}$, we obtain

$$
\begin{aligned}
& \left|f\left(\frac{a+b}{2}\right)-\frac{\Gamma(\alpha+1)}{(b-a)^{\alpha} 2^{1-\alpha}}\left[J_{\left(\frac{a+b}{2}\right)^{\alpha}}^{\alpha} f(a)+J_{\left(\frac{a+b}{2}\right)^{+}}^{\alpha}(b)\right]\right| \\
& \leq \frac{(b-a)^{2}}{8(\alpha+1)(\alpha+2)^{1-\frac{1}{q}}}\left\{\left(\frac{(\alpha+2)\left|f^{\prime \prime}\left(\frac{a+b}{2}\right)\right|^{q}+\left|f^{\prime \prime}(a)\right|^{q}}{\alpha+3}\right)^{\frac{1}{q}}\right. \\
& \left.\quad+\left(\frac{(\alpha+2)\left|f^{\prime \prime}\left(\frac{a+b}{2}\right)\right|^{q}+\left|f^{\prime \prime}(b)\right|^{q}}{\alpha+3}\right)^{\frac{1}{q}}\right\}
\end{aligned}
$$

Remark 2 If we choose $\alpha=1$ in Corollary 2, we have

$$
\begin{aligned}
& \left|f\left(\frac{a+b}{2}\right)-\frac{1}{b-a} \int_{a}^{b} f(x) d x\right| \\
& \leq \frac{(b-a)^{2}}{16 \times 3^{1-\frac{1}{q}}}\left\{\left(\frac{3\left|f^{\prime \prime}\left(\frac{a+b}{2}\right)\right|^{q}+\left|f^{\prime \prime}(a)\right|^{q}}{4}\right)^{\frac{1}{q}}+\left(\frac{3\left|f^{\prime \prime}\left(\frac{a+b}{2}\right)\right|^{q}+\left|f^{\prime \prime}(b)\right|^{q}}{4}\right)^{\frac{1}{q}}\right\} .
\end{aligned}
$$


Theorem 5 Let $f:[a, b] \rightarrow \mathbb{R}$ be twice differentiable mapping on $(\mathrm{a}, \mathrm{b})$ with $0 \leq \mathrm{a}<\mathrm{b}$. If $\left|\mathrm{f}^{\prime \prime}\right|^{\mathrm{q}}$ is convex on $[\mathrm{a}, \mathrm{b}]$ for same fixed $\mathrm{q}>1$, then the following inequality for fractional integrals holds:

$$
\begin{aligned}
& \mid(\alpha+1)(1-\lambda)^{\alpha} \lambda^{\alpha} f(\lambda a+(1-\lambda) b)-\frac{(\alpha+1) \Gamma(\alpha+1)}{(b-a)^{\alpha}} \\
& \quad \times\left[\lambda^{\alpha+1} J_{(\lambda a+(1-\lambda) b)^{-}}^{\alpha} f(a)+(1-\lambda)^{\alpha+1} J_{(\lambda a+(1-\lambda) b)^{+}}^{\alpha} f(b)\right] \mid \\
& \leq \frac{(b-a)^{2}(1-\lambda)^{\alpha+1} \lambda^{\alpha+1}}{(p(\alpha+1)+1)^{\frac{1}{p}}}\left\{(1-\lambda)\left(\frac{\left|f^{\prime \prime}(\lambda a+(1-\lambda) b)\right|^{q}+\left|f^{\prime \prime}(a)\right|^{q}}{2}\right)^{\frac{1}{q}}\right. \\
& \left.\quad+\lambda\left(\frac{\left|f^{\prime \prime}(\lambda a+(1-\lambda) b)\right|^{q}+\left|f^{\prime \prime}(b)\right|^{q}}{2}\right)^{\frac{1}{q}}\right\} .
\end{aligned}
$$

where $\frac{1}{p}+\frac{1}{q}=1, \lambda \in(0,1)$ and $\alpha>0$.

Proof. Using Lemma 3, convexity of $\left|\mathrm{f}^{\prime \prime}\right|^{q}$ well-known Hölder's inequality, we have

$$
\begin{aligned}
& \mid(\alpha+1)(1-\lambda)^{\alpha} \lambda^{\alpha} f(\lambda a+(1-\lambda) b)-\frac{(\alpha+1) \Gamma(\alpha+1)}{(b-a)^{\alpha}} \\
& \quad \times\left[\lambda^{\alpha+1} J_{(\lambda a+(1-\lambda) b)-}^{\alpha} f(a)+(1-\lambda)^{\alpha+1} J_{(\lambda a+(1-\lambda) b)+}^{\alpha} f(b)\right] \mid \\
& \leq(b-a)^{2}(1-\lambda)^{\alpha+1} \lambda^{\alpha+1}\left\{(1-\lambda)\left(\int_{0}^{1} t^{p(\alpha+1)}\right)^{\frac{1}{p}}\right. \\
& \left(\int_{0}^{1}\left|f^{\prime \prime}[t(\lambda a+(1-\lambda) b)+(1-t) a]\right|^{q} d t\right)^{\frac{1}{q}} \\
& \left.\quad+\lambda\left(\int_{0}^{1}(1-t)^{p(\alpha+1)}\right)^{\frac{1}{p}}\left(\int_{0}^{1}\left|f^{\prime \prime}[t b+(1-t)(\lambda a+(1-\lambda) b)]\right|^{q} d t\right)^{\frac{1}{q}}\right\} \\
& \left.\leq(b-a)^{2}(1-\lambda)^{\alpha+1} \lambda^{\alpha+1}\right\} \\
& \quad \times\left\{(1-\lambda) \frac{1}{(p(\alpha+1)+1)^{\frac{1}{p}}}\left(\int_{0}^{1}\left[t\left|f^{\prime \prime}(\lambda a+(1-\lambda) b)\right|^{q}+(1-t)\left|f^{\prime \prime}(a)\right|^{q}\right] d t\right)^{\frac{1}{q}}\right.
\end{aligned}
$$




$$
\begin{aligned}
& \left.+\lambda \frac{1}{(p(\alpha+1)+1)^{\frac{1}{p}}}\left(\int_{0}^{1}\left[t\left|f^{\prime \prime}(b)\right|^{q}+(1-t)\left|f^{\prime \prime}(\lambda a+(1-\lambda) b)\right|^{q}\right] d t\right)^{\frac{1}{q}}\right\} \\
& =\frac{(b-a)^{2}(1-\lambda)^{\alpha+1} \lambda^{\alpha+1}}{(p(\alpha+1)+1)^{\frac{1}{p}}}\left\{(1-\lambda)\left(\frac{\left|f^{\prime \prime}(\lambda a+(1-\lambda) b)\right|^{q}+\left|f^{\prime \prime}(a)\right|^{q}}{2}\right)^{\frac{1}{q}}\right. \\
& \left.+\lambda\left(\frac{\left|f^{\prime \prime}(\lambda a+(1-\lambda) b)\right|^{q}+\left|f^{\prime \prime}(b)\right|^{q}}{2}\right)^{\frac{1}{q}}\right\} .
\end{aligned}
$$

Corollary 3 Under assumption Theorem 5 with $\lambda=\frac{1}{2}$, we obtain

$$
\begin{aligned}
& \left|f\left(\frac{a+b}{2}\right)-\frac{\Gamma(\alpha+1)}{(b-a)^{\alpha} 2^{1-\alpha}}\left[J_{\left(\frac{a+b}{2}\right)^{-}}^{\alpha} f(a)+J_{\left(\frac{a+b}{2}\right)^{+}}^{\alpha} f(b)\right]\right| \\
& \leq \frac{(b-a)^{2}}{8(\alpha+1)(p(\alpha+1)+1)^{\frac{1}{p}}}\left\{\left(\frac{\left|f^{\prime \prime}\left(\frac{a+b}{2}\right)\right|^{q}+\left|f^{\prime \prime}(a)\right|^{q}}{2}\right)^{\frac{1}{q}}\right. \\
& \left.\quad+\left(\frac{\left|f^{\prime \prime}\left(\frac{a+b}{2}\right)\right|^{q}+\left|f^{\prime \prime}(b)\right|^{q}}{2}\right)^{\frac{1}{q}}\right\} .
\end{aligned}
$$

Remark 3 If we choose $\alpha=1$ in Corollary 3, we have

$$
\begin{aligned}
& \left|f\left(\frac{a+b}{2}\right)-\frac{1}{b-a} \int_{a}^{b} f(x) d x\right| \\
& \leq \frac{(b-a)^{2}}{16(2 p+1)^{\frac{1}{p}}}\left\{\left(\frac{\left|f^{\prime \prime}\left(\frac{a+b}{2}\right)\right|^{q}+\left|f^{\prime \prime}(a)\right|^{q}}{2}\right)^{\frac{1}{q}}+\left(\frac{\left|f^{\prime \prime}\left(\frac{a+b}{2}\right)\right|^{q}+\left|f^{\prime \prime}(b)\right|^{q}}{2}\right)^{\frac{1}{q}}\right\} .
\end{aligned}
$$

Theorem 6 Let $f:[a, b] \rightarrow \mathbb{R}$ be twice differentiable mapping on $(a, b)$ with $0 \leq \mathrm{a}<\mathrm{b}$. If $\left|\mathrm{f}^{\prime \prime}\right|^{\mathrm{q}}$ is convex on $[\mathrm{a}, \mathrm{b}]$ for same fixed $\mathrm{q}>1$, then the following inequality for fractional integrals holds:

$$
\begin{aligned}
& \mid(\alpha+1)(1-\lambda)^{\alpha} \lambda^{\alpha} f(\lambda a+(1-\lambda) b)-\frac{(\alpha+1) \Gamma(\alpha+1)}{(b-a)^{\alpha}} \\
& \quad \times\left[\lambda^{\alpha+1} J_{(\lambda a+(1-\lambda) b)^{-}}^{\alpha} f(a)+(1-\lambda)^{\alpha+1} J_{(\lambda a+(1-\lambda) b)^{+}}^{\alpha} f(b)\right] \mid \\
& \leq(b-a)^{2}(1-\lambda)^{\alpha+1} \lambda^{\alpha+1}
\end{aligned}
$$




$$
\begin{aligned}
& \left\{(1-\lambda)\left(\frac{(q(\alpha+1)+1)\left|f^{\prime \prime}(\lambda a+(1-\lambda) b)\right|^{q}+\left|f^{\prime \prime}(a)\right|^{q}}{(q(\alpha+1)+1)(q(\alpha+1)+2)}\right)^{\frac{1}{q}}\right. \\
& \left.+\lambda\left(\frac{(q(\alpha+1)+1)\left|f^{\prime \prime}(\lambda a+(1-\lambda) b)\right|^{q}+\left|f^{\prime \prime}(b)\right|^{q}}{(q(\alpha+1)+1)(q(\alpha+1)+2)}\right)^{\frac{1}{q}}\right\} .
\end{aligned}
$$

where $\lambda \in(0,1)$ and $\alpha>0$.

Proof. Using Lemma 3, convexity of $\left|f^{\prime \prime}\right|^{q}$ well-known Hölder's inequality, we have

$$
\begin{aligned}
& \mid(\alpha+1)(1-\lambda)^{\alpha} \lambda^{\alpha} f(\lambda a+(1-\lambda) b)-\frac{(\alpha+1) \Gamma(\alpha+1)}{(b-a)^{\alpha}} \\
& \quad \times\left[\lambda^{\alpha+1} J_{\left.(\lambda a+(1-\lambda) b)-f(a)+(1-\lambda)^{\alpha+1} J_{(\lambda a+(1-\lambda) b)+f(b)}^{\alpha}\right] \mid}\right. \\
& \leq(b-a)^{2}(1-\lambda)^{\alpha+1} \lambda^{\alpha+1}\left\{(1-\lambda)\left(\int_{0}^{1} 1^{p}\right)^{\frac{1}{p}}\left(\int_{0}^{1} t^{q(\alpha+1)}\left|f^{\prime \prime}[t(\lambda a+(1-\lambda) b)+(1-t) a]\right|^{q} d t\right)^{\frac{1}{q}}\right. \\
& \left.+\lambda\left(\int_{0}^{1} 1^{p}\right)^{\frac{1}{p}}\left(\int_{0}^{1}(1-t)^{q(\alpha+1)}\left|f^{\prime \prime}[t b+(1-t)(\lambda a+(1-\lambda) b)]\right|^{q} d t\right)^{\frac{1}{q}}\right\} \\
& \leq(b-a)^{2}(1-\lambda)^{\alpha+1} \lambda^{\alpha+1}\left\{(1-\lambda)\left(\int_{0}^{1} t^{q(\alpha+1)}\left[t\left|f^{\prime \prime}(\lambda a+(1-\lambda) b)\right|^{q}+(1-t)\left|f^{\prime \prime}(a)\right|^{q}\right] d t\right)^{\frac{1}{q}}\right. \\
& \left.\quad+\lambda\left(\int_{0}^{1}(1-t)^{q(\alpha+1)}\left[t\left|f^{\prime \prime}(b)\right|^{q}+(1-t)\left|f^{\prime \prime}(\lambda a+(1-\lambda) b)\right|^{q}\right] d t\right)^{\frac{1}{q}}\right\} \\
& =(b-a)^{2}(1-\lambda)^{\alpha+1} \lambda^{\alpha+1}\left\{(1-\lambda)\left(\frac{(q(\alpha+1)+1)\left|f^{\prime \prime}(\lambda a+(1-\lambda) b)\right|^{q}+\left|f^{\prime \prime}(a)\right|^{q}}{(q(\alpha+1)+1)(q(\alpha+1)+2)}\right)^{\frac{1}{q}}\right. \\
& \left.\quad+\lambda\left(\frac{(q(\alpha+1)+1)\left|f^{\prime \prime}(\lambda a+(1-\lambda) b)\right|^{q}+\left|f^{\prime \prime}(b)\right|^{q}}{(q(\alpha+1)+1)(q(\alpha+1)+2)}\right)^{\frac{1}{q}}\right\} .
\end{aligned}
$$

Corollary 4 Under assumption Theorem 6 with $\lambda=\frac{1}{2}$, we obtain

$$
\begin{aligned}
& \left|f\left(\frac{a+b}{2}\right)-\frac{\Gamma(\alpha+1)}{(b-a)^{\alpha} 2^{1-\alpha}}\left[J_{\left(\frac{a+b}{2}\right)^{-}}^{\alpha}(a)+J_{\left(\frac{a+b}{2}\right)^{+}}^{\alpha}(b)\right]\right| \\
& \leq \frac{(b-a)^{2}}{8(\alpha+1)}\left\{\left(\frac{(q(\alpha+1)+1)\left|f^{\prime \prime}\left(\frac{a+b}{2}\right)\right|^{q}+\left|f^{\prime \prime}(a)\right|^{q}}{(q(\alpha+1)+1)(q(\alpha+1)+2)}\right)^{\frac{1}{q}}\right.
\end{aligned}
$$




$$
\left.+\left(\frac{(q(\alpha+1)+1)\left|f^{\prime \prime}\left(\frac{a+b}{2}\right)\right|^{q}+\left|f^{\prime \prime}(b)\right|^{q}}{(q(\alpha+1)+1)(q(\alpha+1)+2)}\right)^{\frac{1}{q}}\right\} .
$$

Remark 4 If we choose $\alpha=1$ in Corollary 4, we have

$$
\begin{aligned}
& \left|f\left(\frac{a+b}{2}\right)-\frac{1}{b-a} \int_{a}^{b} f(x) d x\right| \\
& \leq \frac{(b-a)^{2}}{16}\left\{\left(\frac{(2 q+1)\left|f^{\prime \prime}\left(\frac{a+b}{2}\right)\right|^{q}+\left|f^{\prime \prime}(a)\right|^{q}}{(2 q+1)(2 q+2)}\right)^{\frac{1}{q}}\right. \\
& \left.\quad+\left(\frac{(2 q+1)\left|f^{\prime \prime}\left(\frac{a+b}{2}\right)\right|^{q}+\left|f^{\prime \prime}(b)\right|^{q}}{(2 q+1)(2 q+2)}\right)^{\frac{1}{q}}\right\} .
\end{aligned}
$$

\section{References}

[1] S. Belarbi, Z. Dahmani, On some new fractional integral inequalities, $J$. Ineq. Pure and Appl. Math., 10 (3) (2009), Art. 86.

[2] Z. Dahmani, New inequalities in fractional integrals, International Journal of Nonlinear Scinece, 9 (4) (2010), 493-497.

[3] Z. Dahmani, On Minkowski and Hermite-Hadamard integral inequalities via fractional integration, Ann. Funct. Anal., 1 (1) (2010), 51-58.

[4] Z. Dahmani, L. Tabharit, S. Taf, Some fractional integral inequalities, Nonl. Sci. Lett. A, 1 (2) (2010), 155-160.

[5] Z. Dahmani, L. Tabharit, S. Taf, New generalizations of Gruss inequality usin Riemann-Liouville fractional integrals, Bull. Math. Anal. Appl., 2 (3) (2010), 93-99.

[6] S. S. Dragomir, C. E. M. Pearce, Selected Topics on Hermite-Hadamard Inequalities and Applications, RGMIA Monographs, Victoria University, 2000.

[7] R. Gorenflo, F. Mainardi, Fractional calculus: integral and differential equations of fractional order, Springer Verlag, Wien (1997), 223-276. 
[8] S. Hussain, M. I. Bhatti, M. Iqbal, Hadamard-type inequalities for sconvex functions I, Punjab Univ. Jour. of Math., 41 (2009), 51-60.

[9] H. Kavurmaci, M. Avci, M. E. Ozdemir, New inequalities of hermitehadamard type for convex functions with applications, Journal of Inequalities and Applications, 2011, Art No. 86, (2011).

[10] A. A. Kilbas, H. M. Srivastava, J. J. Trujillo, Theory and Applications of Fractional Differential Equations, North-Holland Mathematics Studies, 204, Elsevier Sci. B.V., Amsterdam, 2006.

[11] S. Miller, B. Ross, An introduction to the Fractional Calculus and Fractional Differential Equations, John Wiley \& Sons, USA, 1993, p. 2.

[12] J. E. Pečarić, F. Proschan, Y. L. Tong, Convex Functions, Partial Orderings and Statistical Applications, Academic Press, Boston, 1992.

[13] I. Podlubni, Fractional Differential Equations, Academic Press, San Diego, 1999.

[14] M. Z. Sarikaya, N. Aktan,On the generalization of some integral inequalities and their applications, Mathematical and Computer Modelling, 54, $2175-2182$.

[15] M. Z. Sarikaya, E. Set, M. E. Ozdemir,On some Integral inequalities for twice differantiable mappings, Studia Univ. Babes-Bolyai Mathematica, 59 (1) (2014), 11-24.

[16] M. Z. Sarikaya, A. Saglam, H. Yildirim,New inequalities of HermiteHadamard type for functions whose second derivatives absolute values are convex and quasi-convex, International Journal of Open Problems in Computer Science and Mathematics ( IJOPCM), 5 (3), 2012, 1-14.

[17] M. Z. Sarikaya, H. Ogunmez,On new inequalities via Riemann-Liouville fractional integration, Abstract and Applied Analysis, 2012 Article ID 428983, (2012), 10 pages.

[18] M. Z. Sarikaya, E. Set, H. Yaldiz, N. Basak, Hermite -Hadamard's inequalities for fractional integrals and related fractional inequalities, Mathematical and Computer Modelling, DOI:10.1016/j.mcm.2011.12.048, 57 (2013) 2403-2407. 
[19] M. Z. Sarikaya, H. Yildirim, On Hermite-Hadamard type inequalities for Riemann-Liouville fractional integrals, Submited

[20] M. Tunc, On new inequalities for $\mathrm{h}$-convex functions via RiemannLiouville fractional integration, Filomat 27:4 (2013), 559-565.

[21] J. Wang, X. Li, M. Feckan, Y. Zhou, Hermite-Hadamard-type inequalities for Riemann-Liouville fractional integrals via two kinds of convexity, Appl. Anal. (2012). doi:10.1080/00036811.2012.727986.

[22] B-Y, Xi, F. Qi, Some Hermite-Hadamard type inequalities for differentiable convex functions and applications, Hacet. J. Math. Stat., 42 (3) (2013), 243-257.

[23] B-Y, Xi, F. Qi, Hermite-Hadamard type inequalities for functions whose derivatives are of convexities, Nonlinear Funct. Anal. Appl., 18 (2) (2013), $163-176$.

[24] Y. Zhang, J-R. Wang, On some new Hermite-Hadamard inequalities involving Riemann-Liouville fractional integrals, Journal of Inequalities and Applications, 2013:220, (2013). 